\title{
Factors affecting resumption of forest or satoyama usage by former evacuees following their return after the Fukushima Dai-ichi Nuclear Power Plant accident
}

\author{
M. Takada ${ }^{1,2}$, T. Yasutaka ${ }^{2, a, *}$, Y. Kanai ${ }^{2}$ and Y. Kuroda ${ }^{3}$ \\ ${ }^{1}$ National Institutes for Quantum and Radiological Science and Technology, Chiba-city, Chiba, Japan. \\ 2 National Institute of Advanced Industrial Science and Technology, Tsukuba-City, Ibaraki, Japan. \\ ${ }^{3}$ Fukushima Prefectural Centre for Environmental Creation, Miharu-town, Fukushima, Japan.
}

Received: 27 October 2020 / Accepted: 23 November 2020

\begin{abstract}
In the rural areas of Fukushima, many residents participate in a culture of satoyama usage, and this cultural practice should be respected when conducting radiation protection. We conducted a case study by performing interview survey and text analysis of former evacuees in a rural part of a former evacuation area in Fukushima, to evaluate their attitude towards personal usage of forest or satoyama after radiocaesium contamination and long evacuation following the Fukushima Dai-ichi nuclear accident in 2011. The evacuation order in the study area was lifted in 2017, and our interview survey was conducted in 2019. We found that the resumption to the use of satoyama after their return varied among residents in this particular area. Most returnees in the area were highly knowledgeable about radiation in the environment, but we could not conclude that it helped them make decisions regarding whether to resume satoyama usage. Residents resuming personal use of satoyama after their return were those who had a close relationship with satoyama before the accident, such as the use of forest products for commercial purpose. These past experiences may have led them to have positive attitudes towards the resumption of satoyama usage after their return. Finally, we identified the required approaches towards resuming satoyama usage based on the interviewees' different attitudes.
\end{abstract}

Keywords: stakeholder involvement / quality of life / co-expertise process / interview survey

\section{Introduction}

Environmental pollution caused by a nuclear accident affects various aspects of life for residents in contaminated areas. It is not only the environment, which is directly affected, but also the social and economic aspects and the quality of life of the residents. On the other hand, in the recovery process of contaminated areas, the key issue is to maintain their quality of life in this process (Dubreuil et al., 1999). The Fukushima Daiichi Nuclear Power Plant accident in 2011 caused widespread radioactive contamination. Approximately $71 \%$ of the Fukushima Prefecture is forest (Yoshihara et al., 2014), and the majority of the forest area is a traditional Japanese rural landscape called satoyama (Biodiversity Center of Japan, http://www.biodic.go.jp/ne_research_e.html). The Japanese term "satoyama" was originally used to indicate an agricultural forest, and the majority of satoyama comprise secondary forest, including coppice woodlands and pine forests

*Corresponding author: t.yasutaka@aist.go.jp
(Takeuchi 2001), that has been affected by human activities. People in Japan have benefited in various ways from satoyama for a long time (Takeuchi et al., 2016), such as sales of timber and mushroom logs. In addition, satoyama is not only a source of income for rural residents but also an important resource for their quality of life (Takeuchi 2001). For example, people traditionally collect fallen leaves from the forest and ferment them for use as agricultural compost (Miyaura, 2009). Furthermore, the collection and consumption of wild foods, including not only game animals and mushrooms, but also edible wild plants (sprouts of wild plants and trees), form an important part in the residents' traditional cultures (Kiyono and Akama, 2013; Tagami and Uchida 2017). The need to take cultural considerations into account in protecting people's quality of life has previously been discussed in the field of radiation protection. In Norway, after the Chernobyl accident in 1986, traditional reindeer herding was preserved. This decision was made from the perspective of protecting both cultural and socio-economic aspects, in a system of vigilance towards radioactive materials in the environment (Liland et al., 2010). The culture of satoyama usage in rural areas in 
Fukushima, therefore, must be considered from a similar perspective. In the event that local residents wish to maintain their traditional culture of satoyama usage to improve their quality of life, promoting informed decision-making by the residents about satoyama usage including vigilance towards the presence of radiation in satoyama is important.

In general, due to their large surface area, satoyama or forests are more difficult to decontaminate and prevent further radioactive contamination than other areas, such as farmlands and residential areas. In contaminated forests, it is known that radioactive materials circulate within a forest through plant roots, leaves, and soils (Shaw 2007). After the Chernobyl accident in 1986, it was noted that the contamination of forest products, such as wild foods, was prolonged (IAEA, 2006, 2010). In Japan, after the Fukushima accident, some commonly consumed edible wild plants and mushrooms showed a tendency to have high levels of radiocaesium (Yamaji et al., 2016; Komatsu et al., 2019). The Ministry of Agriculture, Forestry and Fisheries (MAFF) reported that few agricultural products, such as beans, vegetables, and fruits, have been found to exceed the food standard of $100 \mathrm{~Bq} / \mathrm{kg}$ (Ministry of Health, Labour and Welfare (MHLW), https:// www.mhlw.go.jp/english/topics/2011eq/dl/food-120821_1. pdf) since 2015 , while wild foods such as edible wild plants and mushrooms have been found to exceed the standard as of 2020 (MAFF, https://www.maff.go.jp/e/policies/food safety/ h30gaiyo.html).

Although many evacuees have been returning to their homes in the former evacuation area in Fukushima after the decontamination and the decrease in the air dose rate, most of the forests in the area are still contaminated because of the circulation of radiocaesium. The countermeasures in forested areas implemented in Fukushima were the removal of the organic layer from the forest floor within an area of approximately $20 \mathrm{~m}$ from frequently used areas, such as residential areas and farmlands. This was mainly performed to reduce the air dose rate around and within frequently used areas and not in forests (Ministry of the Environment, 2015).

There are regulations on forest products. Forest management activities and shipments of timber are prohibited at $2.5 \mu \mathrm{Sv} / \mathrm{h}$ or higher, shipment of mushroom logs is less than $50 \mathrm{~Bq} / \mathrm{kg}$ (Forestry Agency, 2018), and agricultural use of compost made from fallen leaves is less than $400 \mathrm{~Bq} / \mathrm{kg}$ (MAFF, https://www.maff.go.jp/e/policies/food_safety/emer/ attach/pdf/201704_summary.pdf). Regulations for wild foods, such as game meat, mushrooms, and edible wild plants, are considered to be the same as general market foods, with shipping restrictions at $100 \mathrm{~Bq} / \mathrm{kg}$ or more; personal consumption is not prohibited.

To resume the commercial use of forests, the Forestry Agency and Fukushima Prefecture are leading a demonstration project to reduce radiation exposure to workers during operations and to develop a system to supply safe wood products (Forestry Agency, 2018). However, for personal satoyama usage as a precious cultural aspect in rural area, such as the collection and consumption of wild foods and the use of fallen leaves for composting, no support such as a demonstration project for commercial forestry have been imposed except for those regulations. To promote informed decision-making towards local residents' resumption of personal satoyama usage, in addition to considering vigilance to the presence of radiation, it is necessary to investigate residents' attitudes regarding satoyama usage and examine relevant issues in the areas affected by the nuclear disaster.

This study focused on the personal use of satoyama after the Fukushima accident in one of the former evacuation areas, and the factors influencing residents who expressed positive or negative attitudes on resumption after their return were examined; focus was also on those who had already resumed satoyama usage. An interview survey was conducted for residents of the Yamakiya district in Kawamata town. Then, the required approaches for the residents who showed positive attitudes to resume satoyama usage were identified.

\section{Methods}

\subsection{Study area}

The study site was the Yamakiya district, Kawamata town, Fukushima Prefecture, about $40 \mathrm{~km}$ northwest of the Fukushima Dai-ichi nuclear power plant. The main industry before the accident was agriculture, involving tobacco, flower, and rice cultivation (Award, 1988; Yasutaka et al., 2020). The population in March 2011, just before the accident, was about 1300 (Kawamata town, https://www.town.kawamata.lg.jp/site/sinsaisaigai/yamakiyatikukyojyuujyoukyou.html). After the accident, only the Yamakiya district in Kawamata town was designated as a deliberate evacuation area in April 2011, and residents were evacuated from this area. Decontamination was conducted in this district from March to December 2015 during evacuation of the residents for about 1600 ha of residential and agricultural areas, roads, and areas $20 \mathrm{~m}$ from the forest edge (Ministry of the Environment, http://josen.env.go.jp/area/details/kawamata. html). On 31 March 2017, the evacuation order was lifted. As of 1 July 2020, approximately 350 people have returned (Kawamata town, https://www.town.kawamata.lg.jp/site/sin sai-saigai/yamakiyatikukyojyuujyoukyou.html), and many of them are elderly (Yasutaka et al., 2020). Currently, the production of flowers has recovered to the level before the accident, and rice farming has also resumed aggressively.

An airborne monitoring survey of radioactive contamination in the Yamakiya district showed that the geometric mean of radiocaesium $\left({ }^{134} \mathrm{Cs}\right.$ and $\left.{ }^{137} \mathrm{Cs}\right)$ deposition in the district was $263 \mathrm{kBq} / \mathrm{m}^{2}$ in a range of $200-2000 \mathrm{kBq} / \mathrm{m}^{2}$ as of 28 June 2012 (Fifth Airborne Monitoring Survey by MEXT, https:/emdb. jaea.go.jp/emdb/en/portals/b1020201/). On 16 November 2017, air dose rate $1 \mathrm{~m}$ above the ground, after decontamination and lifting of evacuation order, was $0.59 \mu \mathrm{Sv} / \mathrm{h}(0.23-$ $2.2 \mu \mathrm{Sv} / \mathrm{h}$ ) (Twelfth Airborne Monitoring Survey by the Secretariat of the Nuclear Regulation Authority, https://emdb. jaea.go.jp/emdb/en/portals/b1010301/). The air dose rate in the region is displayed with land-use in Figure 1 (Ministry of Land, Infrastructure and Transport, https://nlftp.mlit.go.jp/ksj/ gml/datalist/KsjTmplt-L03-b.html\#!). The geometric mean of air dose rate in forested area was $0.62 \mu \mathrm{Sv} / \mathrm{h}(0.25-2.2 \mu \mathrm{Sv} / \mathrm{h})$, and that of other areas (e.g. agricultural land, residential area, abandoned land) was $0.53 \mu \mathrm{Sv} / \mathrm{h}(0.23-1.8 \mu \mathrm{Sv} / \mathrm{h})$. Currently, people, including the local residents in the district, can enter the entire forest freely, as with other land-use areas. No personal activities in satoyama are prohibited, although there are still some regulations for commercial forestry, as mentioned previously. 

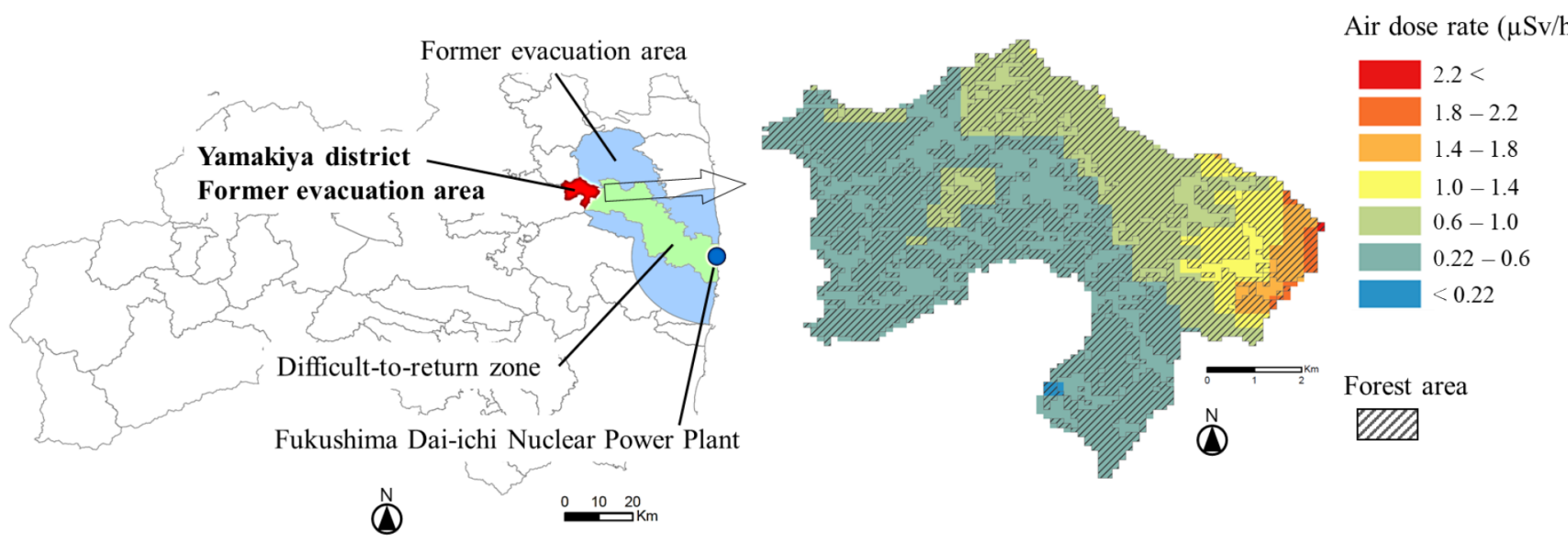

Fig. 1. Location of the present study site (left) and map showing the air dose rate $1 \mathrm{~m}$ above the ground on 16 November 2017 , alongside land use in the region.

Table 1. Interviewee information and responses on Satoyama usage.

\begin{tabular}{|c|c|c|c|c|c|c|}
\hline \multirow[t]{3}{*}{ ID } & \multirow[t]{3}{*}{ Gender } & \multirow[t]{3}{*}{ Age } & \multicolumn{4}{|c|}{ Situation of Satoyama usage } \\
\hline & & & \multicolumn{2}{|c|}{ Consumption status of edible wild plants } & \multirow{2}{*}{$\begin{array}{l}\text { Sale of timber before } \\
\text { the accident }\end{array}$} & \multirow{2}{*}{$\begin{array}{l}\text { Composting of fallen leaves } \\
\text { for agricultural use }\end{array}$} \\
\hline & & & $\begin{array}{l}\text { Before the } \\
\text { accident }\end{array}$ & $\begin{array}{l}\text { After the } \\
\text { accident }\end{array}$ & & \\
\hline $\mathrm{a}$ & Male & $60-69$ & Yes & No & No & No \\
\hline $\mathrm{b}$ & Male & $60-69$ & Yes & Yes & Yes & Yes \\
\hline d & Male & $30-39$ & No & No & No & No \\
\hline $\mathrm{e}$ & Female & $50-59$ & Yes & No & No & Yes \\
\hline$f$ & Male & $70-79$ & Yes & Yes & Yes & Yes \\
\hline g & Male & $60-69$ & Yes & Yes & No & - \\
\hline $\mathrm{h}$ & Female & $70-79$ & Yes & Yes & No & - \\
\hline $\mathrm{i}$ & Male & $60-69$ & Yes & No & No & No \\
\hline
\end{tabular}

The activity concentrations of radiocaesium in wild foods collected from the forest in the district were measured in 2018. Udo (edible wild plant, Aralia cordata) showed $<1.9$ and $6.8 \mathrm{~Bq} / \mathrm{kg}$-wet for ${ }^{134} \mathrm{Cs}$ and ${ }^{137} \mathrm{Cs}$, respectively. For koshiabura (edible wild plant, Chengiopanax sciadophylloides), ${ }^{134} \mathrm{Cs}$ was $234 \mathrm{~Bq} / \mathrm{kg}$-wet and ${ }^{137} \mathrm{Cs}$ was $2224 \mathrm{~Bq} / \mathrm{kg}$ wet (unpublished data). Yamaji et al. (2016) also reported high concentration of radiocaesium in koshiabura. For reference, spinach grown on decontaminated farmland in 2018 was $<1.3$ and $1.2 \mathrm{~Bq} / \mathrm{kg}$-wet for ${ }^{134} \mathrm{Cs}$ and ${ }^{137} \mathrm{Cs}$, respectively and watercress showed $<1.6 \mathrm{~Bq} / \mathrm{kg}$-wet for ${ }^{134} \mathrm{Cs}$ and $4.5 \mathrm{~Bq} / \mathrm{kg}$-wet for ${ }^{137} \mathrm{Cs}$ (unpublished data). Accordingly, in the study region, informed decision-making by local residents towards the resumption of satoyama usage, such as the consumption of edible wild plants, is possible, provided that there is appropriate vigilance regarding the presence of radiation.

\subsection{Interview survey}

We conducted an interview survey from May to June 2019 in Yamakiya district on the local residents who returned to the district and regarding their use of forests and agricultural fields. Environmental surveys were conducted in the district, with the cooperation of the local residents, and in doing so, relationships were developed between them and the authors. This relationship was developed through cooperation in activities, including working together on farming activities, performing radioactivity measurements of residents' agricultural products, and conducting study workshops regarding radiation in the environment (Schneider et al., 2019; Yasutaka et al., 2020). In this study, we conducted the interviews to survey the residents' views on these relationships. Ten interviewees were selected according to the age structure of the district, gender, and usage of satoyama (Tab. 1). Two interviewers conducted the survey for 30 minutes to one hour per resident. The interview survey covered:

- perceptions of the current situation in the district compared to the situation before the accident;

- attitudes towards radiation in contaminated forests and satoyama usage;

- attitudes towards decontamination in agricultural fields;

- change in quality of life. 
Table 2. Topic codes and related words in the interview.

\begin{tabular}{|c|c|c|c|}
\hline Topic code & $\begin{array}{l}\text { Words related to } \\
\text { topic code }\end{array}$ & Remarks & \\
\hline \multirow[t]{2}{*}{$\begin{array}{l}\text { (A) Scientific knowledge } \\
\text { on radiation }\end{array}$} & $\begin{array}{l}\text { (A-1) Effect on a } \\
\text { human body }\end{array}$ & $\begin{array}{l}\text { Radiation (放射線), risk (リスク), safety (安全), } \\
\text { danger (危険), radioactivity (放射能) }\end{array}$ & \\
\hline & $\begin{array}{l}\text { (A-2) Radiation in } \\
\text { the environment }\end{array}$ & $\begin{array}{l}\text { Data (デー夕), value (数值), cycle (循環), } \\
\text { measurement (測定), Bq (ベクレル), } \\
\text { radiation (放射), dose (線量) }\end{array}$ & \\
\hline & (B-2) Sale of timber & Logs (原木), logging (伐採) & $\begin{array}{l}\text { Experience to obtain income } \\
\text { through sale of timbers }\end{array}$ \\
\hline & (B-3) Wild foods & $\begin{array}{l}\text { Shiitake mushroom (シイタケ), mushroom } \\
\text { (キノコ), edible wild plant (山菜) }\end{array}$ & \\
\hline (C) Natural hazard & & Hazard (災害), heavy rain (大雨) & $\begin{array}{l}\text { Natural hazard such as landslide } \\
\text { related to intensive decontamination } \\
\text { or reducing frequency of forest } \\
\text { usage due to non-decontamination }\end{array}$ \\
\hline (E) Forest decontamination & & $\begin{array}{l}\text { Remove (取り除く), forest decontamination } \\
\text { (山の除染, 山林除染, 森林除染), } \\
\text { nakedness (丸裸), living space (生活圏) }\end{array}$ & \\
\hline
\end{tabular}

The contents of the survey were referenced from a previous study (Yasutaka et al., 2020) and prepared with a specialist in qualitative research. As the procedure of the survey, the main interviewer orally described an overview of the survey and handling of personal information, and obtained signed consent to participate in the survey. Semi-structured interviews were adopted using the interview guide (Appendix) described above.

Satoyama usage in the district before the accident includes consumption of edible wild plants and mushrooms, sale of timber, and composting with fallen leaves for agriculture. However, none of the interviewees resumed those activities after their return, except personal consumption of edible wild plants. Therefore, in the present study, resumption of satoyama usage after return is focused on the personal consumption of edible wild plants.

\subsection{Analysis}

The interview survey was transcribed and only texts from interviewees were targeted for the analysis. Words for the analysis (nouns, verbs, adverbs, and adjectives) were extracted using the text data analysis tool KH Corder (Higuchi, 2016, 2017). "Decontamination (josen)" and "living area (seikatuken)" were manually extracted because it was not performed automatically, thus affecting the results of analysis. A cooccurrence network of words with similar patterns of occurrence was illustrated for automatically extracted words, and the lines connecting words with similar patterns of occurrence classified topics in the interviews. The Jaccard coefficient was used as a measure of the degree of cooccurrence. In addition, we selected eight topic codes that were expected to affect attitudes towards satoyama usage after resident return (Tab. 2). Two topic codes were about scientific knowledge of residents on radiation (A): "radiation effect on a human body" (A-1) and "radiation in the environment" (A-2). Three topic codes were about relation of residents with satoyama or forest (B): "composting of fallen leaves" (B-1), "sale of timber" (B-2), and "wild foods" (B-3). Other topic codes were "natural hazards" related to intensive forest decontamination or reducing frequency of forest usage due to non-decontamination (C), "damage by animal" related to changes in the frequency of forest usage (D), and "forest decontamination" (E). Words related to each topic code in the interviews were listed by reviewing the transcripts. The texts were coded according to the presence or absence of related words. Correspondence analysis was conducted to evaluate the similarity of the patterns of the topic code occurrence by satoyama usage of the residents and to visualise them in two dimensions. A short distance between topic codes indicates that these are used close together in sentences and that between-group labels indicate their similarities. Codes that appear on the opposite side of the contrasting group are characteristic of the target group. The interviews and analyses were conducted in Japanese and then translated into English.

\section{Results}

\subsection{Properties of interviewees}

Following the interviews, the current status of satoyama usage after resident return was classified into three groups: three residents did not voluntarily collect and consume edible wild plants even before the accident ("never use group"), but they had experiences consuming edible wild plants collected 
Table 3. List of the 25 most frequent words.

\begin{tabular}{llll}
\hline Words & Freq. & Words & Freq. \\
\hline Man (人) & 515 & Month (月) & 136 \\
Think (思う) & 300 & Mountain (山) & 135 \\
Now (今) & 260 & I (自分) & 129 \\
Say (言う) & 254 & Disaster (震災) & 108 \\
Year (年) & 235 & After all (結局) & 105 \\
Go (行く) & 224 & Talk (話) & 100 \\
Come (来る) & 214 & Town (町) & 91 \\
Understand (分かる) & 195 & See (見る) & 87 \\
Previous (前) & 190 & Child (子ども) & 80 \\
Come out (出る) & 144 & Decontamination (除染) & 79 \\
Evacuation (避難) & 141 & Vary (違う) & 78 \\
Enter (入る) & 140 & Return (帰る) & 78 \\
After (あと) & 138 & & \\
\hline
\end{tabular}

by their family living together; three residents used to consume edible wild plants before the accident, but have been avoiding using them after their return ("avoiding use group"), four residents used to consume edible wild plants before the accident and have resumed their use after their return ("resuming use group"). None of the residents who did not collect and consume edible wild plants started deliberately consuming them after the accident.

\subsection{Frequent words and topics in the interview}

The total number of sentences by 10 interviewees was 6,259 . Table 3 shows the top 25 words. Distinctive words were "understand" (195 times), "evacuation" (141 times), "mountain" (135 times), "disaster" (108 times), "child" (80 times), "decontamination" (79 times), and "return" (78 times)". Common words such as "think" and "say" are likely to appear frequently in any text (Higuchi, 2016).

The co-occurrence network among 103 words limited to nouns used 15 or more times is shown in Figure 2. KH Coder generates a network by connecting 60 pairs of the most strongly co-occurring words by lines. The network indicates six major topics in the interview:

- situation of Yamakiya district before the accident;

- agriculture and satoyama usage before and after the accident;

- evacuation and life at temporary houses;

- radiation and radioactivity;

- administrative actions at the accident;

- decontamination in forest and farmland.

An example reply to the "agriculture and satoyama usage before and after the accident" question was "Before the accident I went to forest far more often during mushrooms and edible wild plant seasons in fall and early spring." (resuming use, b) and "I'm not sure why, but after the accident, going to satoyama to collect edible wild plants became to feel more troublesome." (avoiding use, i). An example reply about "radiation and radioactivity" was I do not worry about the usage of satoyama after the accident. We cannot see radiation." (resuming use, g). Another opinion was" I hate the fact that there is radiation in the environment." (resuming use, b). Examples of responses to "decontamination in forest and farmland" were "I thought forest decontamination was impractical because I heard radiocaesium is circulating in the forest." (never used, c) and "I wanted decontamination for my farmland to remove uncertainty." (never used, d).

\subsection{Topic codes}

Table 4 shows occurrence of each topic in the interview. The most frequent topic code was "radiation in the environment" (A2 ), and appeared 74 times in the interview. The topic code on "wild foods" (B-3), "effect on a human body" (A-1), and "composting fallen leaves" (B-1) appeared 43, 41, and 37 times, respectively. Topic codes on "sale of timber" (B-2), "natural hazard" (C), "damage by animal" (D), and "forest decontamination" (E) appeared 13, 13, 18, and 6 times, respectively.

The results of the correspondence analysis are shown in Figure 3. The unique topic code for the "never use group" was "damage by animals" (D) . For the "avoiding use group", the unique topic codes were "natural hazard" (C) and "forest decontamination" (E). The topic code on "effect on a human body" (A-1) was mainly observed from the "never use group", but the "avoiding use group" also talked about it. The topic code "sale of timber" (B-2) was unique for the "resuming use group". Topic codes "radiation in the environment" (A-2), "wild foods" (B-3), and "composting of fallen leaves" (B-1) were common for the interviewees.

Nine interviewees discussed the most common topic of "radiation in the environment" (A-2). Clear differences in the contents were not observed among the resident groups. Eight of the nine interviewees who discussed 'radiation in the environment' (A-2) talked about experiences to measure radioactivity voluntarily by their own or with someone close to them. An example of such replies is "I have confirmed that our products showed low radioactivity." (never used, d). Scientific information on radiocaesium dynamics obtained in a workshop for residents was also observed: "I've heard that cycle of radiocaesium in the forest has been already fixed and that there is very little runoff into the river." (never used, c).

Most topics on the "effect on a human body" (A-1) were in a negative context, with respect to radiation exposure. Examples were "I'm sure that radiation is dangerous, but I think I should just not go into the contaminated mountain." (never used, d) and "There may be a possibility that effects of radiation exposure could be seen for decades to come." (avoiding use, i).

Topics on "forest decontamination" (E) were unique for the "avoiding use group", and were mostly about the possibility of decontamination. An example was "I wanted to decontaminate the forest in our living area where we used to collect wild foods and for walking." (avoiding use, a). Response to "sale of timber" (B-2) was observed from the "resuming use group", such as "Logs for shiitake mushroom cultivation were shipped all over Japan from this region, and the annual sales of the entire region must have been at least 100 million yen." (resuming use f). The topic on "composting of fallen leaves" (B-1) is also about forest products, but it was a common topic for the residents regardless of groups. An example of response was "I used to collect fallen leaves in autumn and composted 


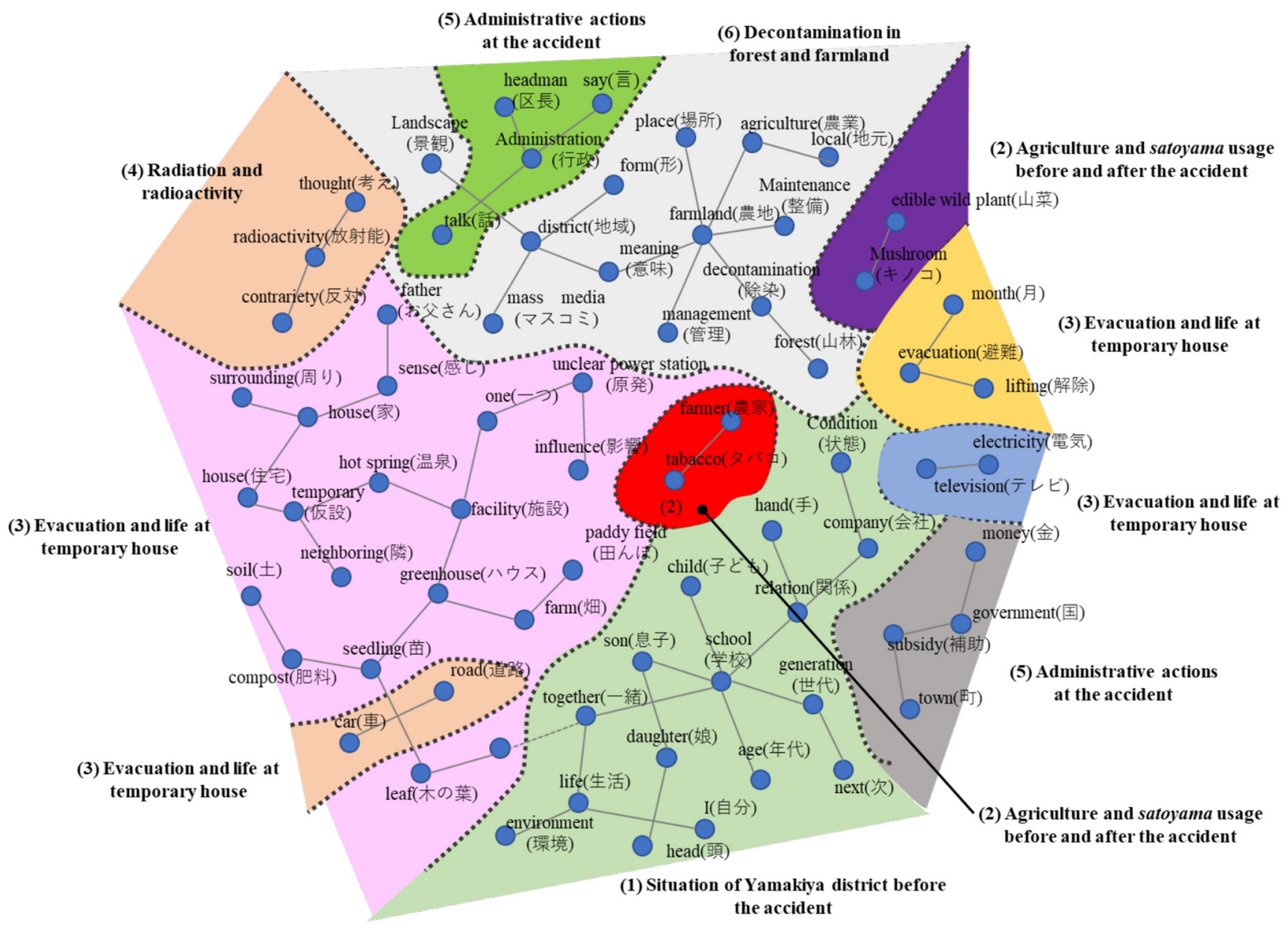

Fig. 2. Co-occurrence network of frequently occurring words. Each group of words connected by dotted lines indicates a cluster of words (topic) used closely in sentences. The following six topics were extracted: (1) situation of Yamakiya district before the accident; (2) agriculture and satoyama usage before and after the accident; (3) evacuation and life at temporary house; (4) radiation and radioactivity; (5) administrative actions at the accident; (6) decontamination in forest and farmland.

Table 4. Cross tabulation of coding results.

\begin{tabular}{lllll}
\hline Topic code & Total & Never use & Avoiding use \\
\hline (A-1) Effect on a human body & 41 & 22 & 13 & Resuming use \\
(A-2) Radiation in the environment & 74 & 27 & 17 & 6 \\
(B-1) Composting fallen leaves & 37 & 11 & 14 & 30 \\
(B-2) Sale of timber & 13 & 2 & 12 & 12 \\
(B-3) Wild foods & 43 & 13 & 6 & 18 \\
(C) Natural hazard & 13 & 2 & 2 & 5 \\
(D) Damage by animal & 18 & 10 & 3 & 6 \\
(E) Forest decontamination & 6 & 3 & 0
\end{tabular}

them in the spring to be used as a tobacco seedbed." (avoiding use, e) and "I need fallen leaves from mountain to improve the soil on farmland." (resuming use f).

\section{Discussion}

We found that in Yamakiya district, some residents used satoyama before the accident and others did not. Some residents who used satoyama before the accident have been avoiding use after the accident, while others resumed using it only for personal consumption of edible wild plants after their return. Here, we discuss the resumption of satoyama usage in relation to scientific information on radiation and past experiences of forest usage. Finally, we identified the required approaches for the residents in Yamakiya who had showed a positive attitude towards resuming the use of satoyama. 


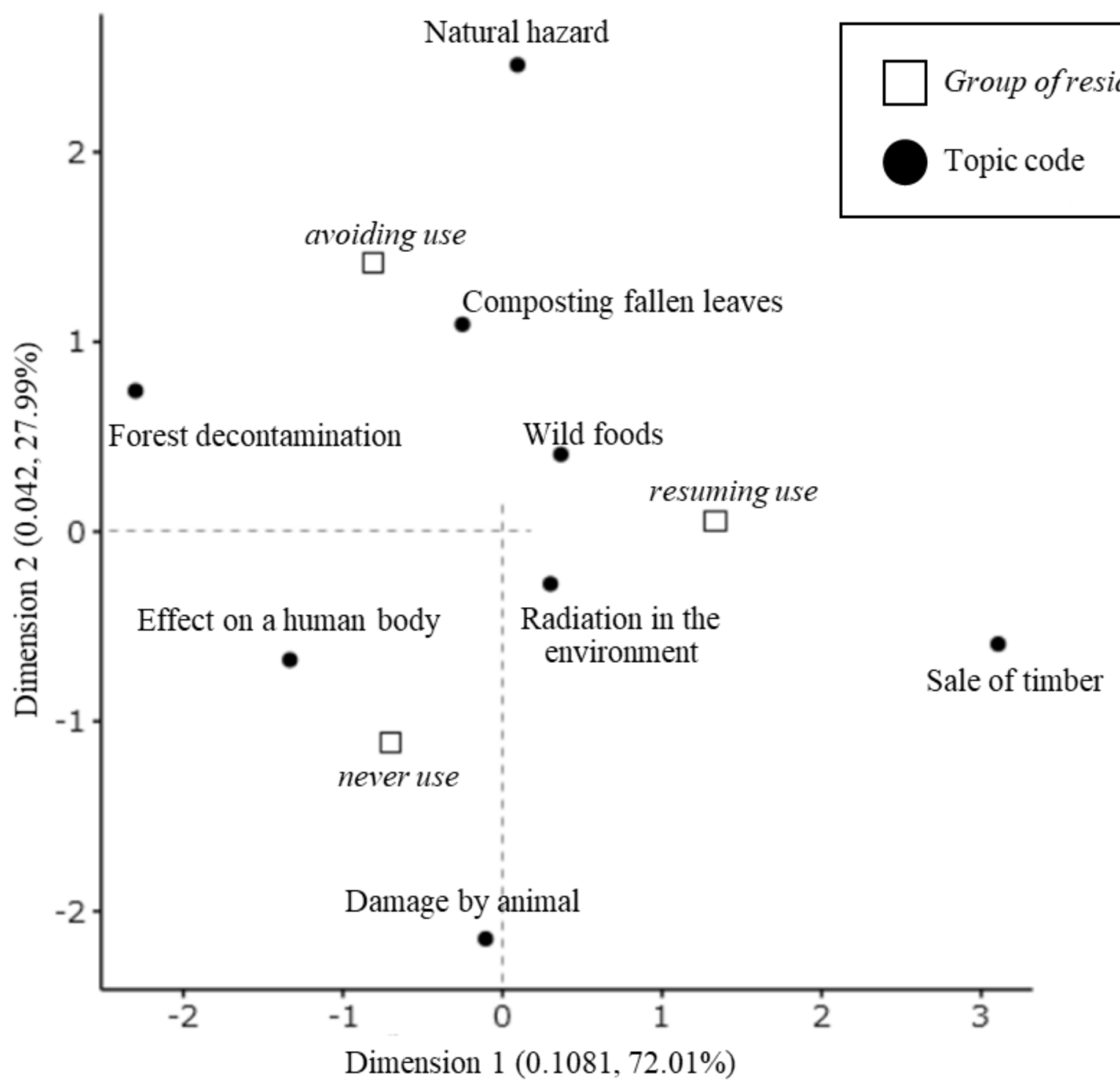

Fig. 3. Correspondence analysis of topic codes: differences by satoyama usage. A short distance between topic codes indicates that these are used close together in sentences and that between-group labels indicate their similarities. Topic codes that appear on the opposite side of the contrasting group are characteristic of the target group.

However, the following two issues should be noted regarding the present results. First, only 10 residents were interviewed. Consequently, the sizes of the analysed texts were limited, and the results of the present study may depend on the selection of the interviewees. Second, this is one case study within a single region, and therefore, the required approaches for the residents are applicable only to the present study site only; the situation may be different in other areas with different contamination levels.

\subsection{Resumption of satoyama usage and scientific knowledge}

Scientific information about radiation risk and environmental conditions are important and fundamental information to decide the individual behaviour (such as satoyama usage) of people residing in contaminated situations. Previous studies have reported that workshops to share knowledge about radiation and activities to measure radiation and share the results help local residents in affected areas understand radiation risk (Yasutaka et al., 2020; Lochard et al., 2020). However, even if people have enough scientific information and understand the risks, they do not always behave in the same way as they did before an accident of that kind.

In this region, exchange between local residents and researchers on radiation and radioactivity has been active since the accident. Researchers have been working together with local residents on farm activities, collecting edible wild plants, and holding study workshops (Yasutaka et al., 2020). This may be one of the reasons why knowledge on radiation and radioactivity was a familiar topic to many residents, and our results suggest that most interviewees understood the approximate contamination level of satoyama products or the air dose rate in satoyama. 
Topics concerning radiation exposure was not observed in the group of residents who resumed using satoyama. The residents in the group were participants of the workshop who shared their knowledge about radiation, and they also cooperated in a radioactivity measurement survey of agricultural products within the district. The workshop and measurement activity may lead to their understanding of radiation risk.

Meanwhile, one group of residents currently avoiding satoyama usage was interested in topics such as a desire for additional forest decontamination and concerns about health due to radiation exposure. These residents were conscious of avoiding radioactive contaminants as much as possible, which may have resulted in them not resuming satoyama usage. In addition, some residents in the group showed loss in motivation in the use of satoyama after their return. Residents in this group may have been affected by a decrease in subjective values for satoyama usage through long-term evacuation, although they did mention that they did not use satoyama because of radiation.

Furthermore, we have to note that a lack of topic concerning radiation do not always indicate that residents are not necessarily worried about radiation. A health consultation project for evacuees of the accident suggested that they tend to hide their fears about the health effects of radiation from others in order to avoid conflicts arising from differences in risk perceptions (Murakami et al., 2017). Accordingly, the group who resumed satoyama usage may have had the possibility to suppress this fear of radiation rather than when there is no concern about usage; consequently, topics on radiation exposure were not observed in the present study.

\subsection{Resumption of satoyama usage and past experiences}

The positive attitudes and decisions to resume satoyama usage were suggested to be related to the experience of using the forest as a source of income or the intensity of involvement with the forest. The group that had already resumed satoyama usage after return included residents with past experience of selling wood. Another commercial use of the forest is the conversion of collected fallen leaves into compost; this experience was, however, not clearly related to satoyama usage after the accident. Even if composted fallen leaves is made for agricultural use, alternative products such as commercial fertilisers are also readily available. On the other hand, the commercial use of the forest through the sale of timber may have strengthened the relationship between the residents and the forest, which may have provided a strong encouragement for resuming satoyama usage after their return. These residents showed a positive attitude towards resuming satoyama usage, and included the topic of resuming the use of fallen leaves as compost.

\subsection{Required approaches and support for each situation}

Resumption to satoyama usage varied among residents in the study area, suggesting that the views of residents who want to use satoyama need to be incorporated into approaches towards resuming this activity, thus emphasizing the importance of stake holder involvement (Schneider et al., 2019). In addition, various attitudes of residents towards resuming satoyama usage after their return indicated that residents are not focused on restoring their lives as before the accident, but are adapting to new living conditions involving radiation standards and risks of radiation exposure, independent of whether the residents themselves are aware of it or not. Yasutaka et al. (2019) insisted on the importance of improving quality of life rather than restoring their lives to how they were before the accident. Accordingly, improving the quality of life of the residents through satoyama usage in new living conditions is important in scenarios where residents want to maintain their traditional culture of satoyama usage. For instance, residents and experts should collaborate to create radiation protection systems as a co-expertise process to promote informed decision-making for local residents, provided that there is vigilance regarding the presence of radiation in the environment (Schneider et al., 2019).

Based on the results of our study, the required approaches and support are summarised for the residents who showed positive attitudes to resuming satoyama usage (Tab. 5). A group of residents who were positive about resuming satoyama usage tended to have a close relationship with the forest. However, their positive attitude towards resumption may not be due to their lower concern about radiation. Therefore, to promote an informed decisionmaking process, including vigilance towards radioactive contamination in satoyama, it is important to create an environment in which residents can easily consult experts about radiation effects on human health and the risk assessment of radiation exposure by satoyama usage. Yasutaka et al. (2020) reported that the co-expertise process in the present study area, such as joint radiation measurements by local residents and experts from outside the area and face-to-face feedback of the results, may lead to decreased anxiety among the local residents. To recover the purposes and frequency of satoyama usage to pre-accident levels, it would be effective to consult and work together on demonstrating the use of satoyama with experts on the fate of radioactive materials in the environment.

We do not recommend that residents who currently show negative attitudes towards resuming satoyama usage should resume. However, there is a possibility that they may change their mind regarding resumption, as some of them had used it before the accident if usage spreads within their community. In cases where these residents become interested in resuming satoyama usage, the same process is required for residents who are positive about resuming satoyama usage, as discussed above. For residents who were not interested in using satoyama initially, there was no problem with the current situation. However, given the possibility that they could be interested in satoyama usage in the future, it is necessary that information are shared equally within the community.

This study is one case study and is limited by the small number of interviewees. However, this is the first interview on such a subject in the former evacuation area of Fukushima, involving the factors affecting residents' attitudes towards resuming satoyama usage in the contaminated area. 
Table 5. Approaches for attitudes of residents.

\begin{tabular}{ll}
\hline $\begin{array}{l}\text { Attitude towards resuming } \\
\text { satoyama usage }\end{array}$ & Required approach \\
\hline Positive & $\begin{array}{l}\text { - To have a close relationship with the forest before the accident } \\
\text { - To create relationships and interactions between residents and experts for appropriate support } \\
\text { and advice on health and radiation } \\
\text { - To create relationships and interactions between residents and experts for recovery of satoyama } \\
\text { usage to the pre-accident levels } \\
\text { - Not recommended to resume but having a possibility to have positive attitude in the } \\
\text { future if the activities of satoyama usage extend within their community } \\
\text { — In case these residents became interested, the same support is required as for positive attitude } \\
\text { used it before the accident }\end{array}$ \\
Others & $\begin{array}{l}\text { No problem with the current situation, but share the information for future decision } \\
\text { making processes }\end{array}$ \\
\hline
\end{tabular}

\section{Conclusions}

In radiation protection, cultural aspects in a contaminated area need to be respected to protect people's quality of life. In rural areas in Fukushima, the cultural practice of satoyama usage needs to be considered from a similar perspective. In this study, we conducted interviews with 10 residents regarding satoyama usage in 2019 in Yamakiya district, rural areas of Fukushima, where the evacuation order was lifted in 2017. Our survey revealed that scientific information had no clear influence on the decision-making to resume satoyama usage in the region, although it is relatively easy for the residents to obtain scientific information. Some of the residents who used satoyama before the accident resumed using it after returning to the region, while others showed negative attitudes towards resumption. Residents were observed to adapt to new living conditions with a new radiation exposure situation. Some of the residents have taken a positive attitude towards resuming satoyama usage, it is necessary to promote informed decision-making regarding the further expansion of satoyama usage; vigilance is necessary for presence of radiation.

\section{Supplementary material}

Interview guide for interviewers

The Supplementary material is available at https:/www. radioprotection.org/10.1051/radiopro/2020085/olm.

\section{Acknowledgements}

We thank the interviewees in Yamakiya district and the authorities of Kawamata for their assistance during our field survey. We also thank Prof. Kenichi Satoh from Shiga University for his support on text data analysis. This study was supported by JSPS KAKENHI (Grant number 18H04141).

\section{References}

Award U. 1988. Land reclamation project at Miharu-Nanbu area in Fukushima prefecture. J. Irrig. Eng. Rural Plan. 13: 63-66.
Dubreuil GH, Lochard J, Girard P, Guyonnet JF, Le Cardinal G, Lepicard S, Livolsi P, Monroy M, Ollagnon H, Pena-Vega A, Pupin V, Rigby J, Rolevitch I, Schneider T. 1999. Chernobyl post-accident management: the ETHOS project. Health Phys. 77: 361-372.

Forestry Agency. 2018. The current state of radioactive substances and the regeneration of forests and forestry 2018 edition. Japan: Forestry Agency, Ministry of Agriculture, Forestry and Fisheries.

Higuchi K. 2016. A two-step approach to quantitative content analysis: KH coder tutorial using Anne of Green Gables (part I). Ritsumeikan Soc. Sci. Rev. 52: 77-91.

Higuchi K. 2017. A two-step approach to quantitative content analysis: KH coder tutorial using Anne of Green Gables (part II). Ritsumeikan Soc. Sci. Rev. 53: 137-147.

IAEA. 2006. Environmental consequences of the Chernobyl accident and their remediation: twenty years of experience. In: Report of the Chernobyl Forum Expert Group "Environment". Vienna: International Atomic Energy Agency.

IAEA. 2010. Handbook of parameter values for the prediction of radionuclide transfer in terrestrial and freshwater environments. In: Technical Report Series No. 472. Vienna: International Atomic Energy Agency.

Kiyono Y, Akama A. 2013. Radioactive cesium contamination of edible wild plants after the accident at the Fukushima Daiichi Nuclear Power Plant. Jpn. J. For. Environ. 55: 113-118.

Komatsu M, Nishina K, Hashimoto S. 2019. Extensive analysis of radiocesium concentrations in wild mushrooms in eastern Japan affected by the Fukushima nuclear accident: use of open accessible monitoring data. Environ. Pollut. 255: 113236.

Liland A, Oughton D, Bay-Larsen I, Eikelmann IM, Solheim-Hansen H, Skuterud L. 2010. Norwegian testing of the EURANOS framework for post-accident rehabilitation preparedness. Radioprotection 45: S215-S224.

Lochard J, Ando R, Takagi H, Endo S, Momma M, Miyazaki M, Kuroda Y, Kusumoto T, Endo M, Endo S, Koyama Y. 2020. The post-nuclear accident co-expertise experience of the Suetsugi community in Fukushima Prefecture. Radioprotection 55: $225-235$.

Ministry of the Environment. 2015. FY2014 decontamination report - A compilation of experiences to date on decontamination for the living environment conducted by the Ministry of the Environment, Japan. Japan: Ministry of the Environment.

Miyaura T. 2009. Satoyama-a place for preservation of biodiversity and environmental education. Die Bodenkultur 60: 23-29.

Murakami M, Sato A, Matsui S, Goto A, Kumagai A, Tsubokura M, Orita M, Takamura N, Kuroda Y, Ochi S. 2017. Communicating 
with residents about risks following the Fukushima nuclear accident. Asia Pac. J. Public Health 29: 74S-89S.

Schneider T, Maître M, Lochard J, Charron S, Lecomte JF, Ando R, Kanai Y, Kurihara M, Kuroda Y, Miyazaki M. 2019. The role of radiological protection experts in stakeholder involvement in the recovery phase of post-nuclear accident situations: some lessons from the FukushimaDaïchi NPP accident. Radioprotection 54: 259-270.

Shaw G. 2007. Radionuclides in forest ecosystems. Radioact. Environ. 10: 127-155.

Tagami K, Uchida S. 2017. Changes of effective half-lives of $137 \mathrm{Cs}$ in three herbaceous plants and bioavailable $137 \mathrm{Cs}$ fraction in soil after the Fukushima nuclear accident. Appl. Geochem. 85: 162-168.

Takeuchi K. 2001. Nature conservation strategies for the 'SATOYAMA'and 'SATOCHI', habitats for secondary nature in Japan. Glob. Environ. Res. 5: 193-198.

Takeuchi K, Ichikawa K, Elmqvist T. 2016. Satoyama landscape as social-ecological system: historical changes and future perspective. Curr. Opin. Environ. Sustain. 19: 30-39.

Yamaji K, Nagata S, Haruma T, Ohnuki T, Kozaki T, Watanabe N, Nanba K. 2016. Root endophytic bacteria of a ${ }^{137} \mathrm{Cs}$ and $\mathrm{Mn}$ accumulator plant, Eleutherococcus sciadophylloides, increase ${ }^{137} \mathrm{Cs}$ and $\mathrm{Mn}$ desorption in the soil. J. Environ. Radioactiv. 153: $112-119$.

Yasutaka T, Kondoh A, Kobayashi T, Naito W, Kurihara M, Kanai Y, Kanno G, Hirono A, Kanno A, Ohuchi K. 2019. Dialogue about environmental monitoring and some activities with residents in Yamakiya region. What we should do for the future? In: Proceedings of the international symposium of radiation medical science center of Fukushima Medical University. January 14-15, 2019, Fukushima.

Yasutaka T, Kanai Y, Kurihara M, Kobayashi T, Kondoh A, Takahashi T, Kuroda Y. 2020. Dialogue, radiation measurements and other collaborative practices by experts and residents in the former evacuation areas of Fukushima: a case study in Yamakiya District, Kawamata Town. Radioprotection 55: 215-224.

Yoshihara T, Matsumura H, Tsuzaki M, Wakamatsu T, Kobayashi T, Hashida SN, Nagaoka T, Goto F. 2014. Changes in radiocesium contamination from Fukushima in foliar parts of 10 common tree species in Japan between 2011 and 2013. J. Environ. Radioactiv. 138: $220-226$.

Cite this article as: Takada M, Yasutaka T, Kanai Y, Kuroda Y. 2020. Factors affecting resumption of forest or satoyama usage by former evacuees following their return after the Fukushima Dai-ichi Nuclear Power Plant accident. Radioprotection 55(4): 325-334 